A Mindfulness-Based Intervention for Unaccompanied Refugee Minors:

A pilot study using mixed methods evaluation

Authors: Katleen Van der Gucht ${ }^{1,2}$, Jana Glas ${ }^{1}$, Lucia De Haene ${ }^{1}$, Peter Kuppens ${ }^{1,2}$, Filip Raes ${ }^{1,2}$

\title{
Affiliation of the authors:
}

${ }^{1}$ Leuven Mindfulness Centre, KU Leuven, 3000 Leuven, Belgium

${ }^{2}$ Faculty of Psychology and Educational Sciences, University of Leuven, 3000 Leuven, Belgium

Email addresses: katleen.vandergucht@kuleuven.be, jana.glas@gmail.com, lucia.dehaene@kuleuven.be, peter.kuppens@kuleuven.be, filip.raes@kuleuven.be

Corresponding author: Katleen Van der Gucht, Faculty of Psychology and Educational Sciences, University of Leuven, Tiensestraat 102, 3000 Leuven, Belgium.

Email: katleen.vandergucht@kuleuven.be

Phone: ++32 (0) 16373183

Acknowledgements: This research was supported by a grant from the foundation "Stichting Koningin Paola". Inge De Leeuw and Berti Persoons adapted the mindfulness-based program, with the assistance of Katharina Müllen. Inge De Leeuw and Berti Persoons delivered the intervention. We are grateful to the people of vzw MinorNdako for the organization and their support during the project, and sincerely thank all participants in the study. Special thanks goes to David Lowyck, the chairman of vzw MinorNdako, for his effort in supporting this study. 


\begin{abstract}
Objectives: Unaccompanied refugee minors (UMs) experience worry and rumination, owing to living in refugee shelters and confronting many stressors that range from finances to health problems to personal safety concerns. The purpose of this study was to explore the feasibility of a mindfulness-based intervention (MBI) for UMs residing at the shelters of Minor Ndako in Belgium.

Methods: Of the 34 residents attending an information session, 13 expressed an interest to participate.

Participants were between 13 and 18 years old. A MBI was offered in small groups between March 2015 and July 2016. The impact on symptoms of depression, positive and negative affect and on symptoms of posttraumatic stress was examined using questionnaires. Four individuals participated in a qualitative assessment on how they experienced the mindfulness.

Results: Quantitative findings suggest that a MBI may reduce negative affect and improve positive affect, both with a medium effect size, and reduce symptoms of depression with a large effect size. Qualitative analyses show that experiences are unique and different among participants. Participants who completed the training make use of the mindfulness exercises as a new coping strategy in combination with other familiar coping strategies.
\end{abstract}

Conclusions: Although the feasibility in this population is not straightforward, results suggest that it may be useful to deliver MBIs in refugee shelters for UMs. These results are preliminary and have to be interpreted with caution due to the small self-selected sample. Therefore the results of this study can only be interpreted as initial, and in need of replication.

Keywords: Unaccompanied Refugee Minors, Mindfulness-Based Intervention, mixed methods evaluation, coping, positive and negative affect, depression 
A Mindfulness-Based Intervention for Unaccompanied Refugee Minors: A pilot study with mixed methods evaluation

Unaccompanied refugee minors (UMs) are children and adolescents who have been forced to leave their country without family as a result of violence, deprivation, persecution, or a threat to their lives. They have often been exposed to horrifying traumatic events and often have survived extreme conditions during their flight. Not surprisingly then, empirical studies, reviews, and meta-analyses indicate a high risk of mental health problems among UMs (Derluyn, Mels, \& Broekaert, 2009; Fazel, Reed, Panter-Brick, \& Stein, 2012; Huemer et al., 2009; Lustig et al., 2004) and refugees in general (Fazel, Wheeler, \& Danesh, 2005; Porter \& Haslam, 2005). A metaanalysis including 7000 refugees in western countries indicated that about one in ten refugees has post-traumatic stress disorder (PTSD), about one in 20 has major depression, and about one in 25 has a generalized anxiety disorder, with the probability of comorbidity (Fazel et al., 2005). Longitudinal studies in UMs (Bean, EurelingsBontekoe, \& Spinhoven, 2007; Vervliet, Lammertyn, Broekaert, \& Derluyn, 2014) and adult refugees (Vuković, Jovanović, Kolarić, Vidović, \& Mollica, 2014) reveal that mental health problems (e.g. depression, anxiety and PTSD) persist over time. A 3 year longitudinal study conducted in refugee camps for Bosnian refugees indicated the persistence of psychological problems over time and somatic health problems were more frequent in subjects suffering from mental illness (Vuković et al., 2014).

There is evidence that specialized mental health interventions, delivered in refugee shelters, help to reduce symptoms of mental disorders such as PTSD and depression (Nosè et al., 2017; Tol et al., 2011). However, most studies so far focus on (1) adults (e.g. Acarturk et al., 2016; Neuner et al., 2008; Neuner, Schauer, Klaschik, Karunakara, \& Elbert, 2004) or young children (e.g. Catani et al., 2009), and (2) on targeted interventions for PTSD (Acarturk et al., 2016; Neuner et al., 2008; Neuner et al., 2004). The psychosocial needs of adolescent and young adult refugees remain largely unmet and more age-appropriate interventions are needed (Derluyn \& Broekaert, 2008). Because of the multifaceted nature of the emotional distress involved in this population, it is recommended to use a transdiagnostic approach and to broaden outcomes aside from symptoms of PTSD (see also Miller \& Rasmussen, 2010). Transdiagnostic interventions target a range of symptoms (e.g. stress, anxiety, depression), and aim at impacting common underlying vulnerability factors. A framework provided in a recent study suggest that applying a transdiagnostic approach to PTSD and other trauma-related disorders may be effective in treating these disorders and their related comorbidities (Gutner, Galovski, Bovin, \& Schnurr, 2016). The authors also state that a transdiagnostic approach has the potential to be effectively 
disseminated so that it can be used in places with limited resources. However, the evidence for the transdiagnostic treatment of PTSD is still limited and more studies are needed to understand its effectiveness.

An example of transdiagnostic interventions aimed at remediating several of the psychological processes (e.g. disruptive emotions, rumination and intrusive cognitions) that contribute to emotional distress and PTSD are mindfulness-based approaches. Mindfulness refers to a compassionate and non-judgmental moment-to-moment awareness of one's experiences (Kabat-Zinn, 1994). Two evidence-based interventions to teach mindfulness skills are mindfulness-based stress reduction (MBSR) (Kabat-Zinn, 2013) and mindfulnessbased cognitive therapy (MBCT) (Segal, Williams, \& Teasdale, 2012). Mindfulness-based interventions (MBIs) aim to reduce reactivity to, and avoidance of, internal experiences (cognitions, emotions, physical sensations) and encourage openness and acceptance of these experiences, while encouraging engagement in valued actions (i.e. behaviors that are important to the individual) that may have previously been avoided. MBIs, as defined by Crane et al. (2017) involve extensive training in mindfulness meditation skills and mindfulness meditation practice is employed as a central foundational methodology (Crane et al., 2017). To date there is compelling evidence that MBIs reduce symptoms of stress, anxiety and depression in clinical and non-clinical populations (Khoury et al., 2014; Khoury, Sharma, Rush, \& Fournier, 2015; Kuyken et al., 2016; Spijkerman, Pots, \& Bohlmeijer, 2016). There is also a theoretical rationale for mindfulness modifying pathological processes that maintain PTSD (Lang, 2017). However, the number of studies in this area is small and therefore more research is needed.

To our knowledge, only a few small-scaled qualitative studies have investigated the feasibility of mindfulness exercises when integrated in cognitive behavioral therapy or art therapy for refugees. Three case studies with adult refugees using culturally adapted cognitive behavioral therapy including several aspects of mindfulness, report that the meditation techniques (e.g. body scan and loving kindness meditation) taught during the intervention were well received, tolerated and helpful (Hinton, Ojserkis, Jalal, Peou, \& Hofmann, 2013; Hinton, Pich, Hofmann, \& Otto, 2013; Jalal, Samir, \& Hinton, 2017). Two recent qualitative studies integrating mindfulness and art therapy in a four day group intervention delivered over three weeks to 12 refugees and asylum seekers described how this short intervention meets the needs of this population (Kalmanowitz, 2016; Kalmanowitz \& Ho, 2016). Numerous formal mindfulness meditation exercises were practiced during the intervention and participants reported that the mindfulness meditation exercises helped them to calm down and to improve sleep (Kalmanowitz, 2016). 
Apart from these qualitative reports on interventions that incorporate mindfulness exercises into a more comprehensive treatment, no studies with refugees evaluating the efficacy of a mindfulness training as a standalone intervention have yet been published. Therefore, the question remains whether: an evidence-based intervention such as MBI can be meaningfully applied to support mental well-being in refugees who reside in shelters, where they may experience high levels of ongoing stress and insecurity about the future. In the current study we specifically focus on unaccompanied refugee minors as research with adolescents is scarce and ageappropriate interventions are needed. Previous research in a school and clinical context showed that mindfulness is well-accepted and effective in alleviating emotional distress and reducing cognitive vulnerability factors in adolescents and young adults (Van der Gucht, Takano, Kuppens, \& Raes, 2017; Van der Gucht, Takano, Labarque, et al., 2017; Van der Gucht, Takano, Raes, \& Kuppens, 2018). The present study builds upon this previous research and examines whether it is feasible to deliver an eight week MBI in a sample of unaccompanied foreign minors located in refugee shelters in Belgium (led by a non-profit organization Minor Ndako). The focus of our study is to explore how unaccompanied refugee minors experience a MBI, and what the possible benefits and impediments are of participation. This was studied using both quantitative and qualitative assessment. We hypothesized that our MBI would (1) reduce symptoms of depression, (2) improve positive affect and reduce negative affect, and (3) reduce the impact of traumatic events.

\section{Method}

\section{Participants}

Between March 2015 and July 2016 adolescents and young adult refugees staying at the shelter Minor NDako (Belgium) had the opportunity to participate in mindfulness-based interventions. A first recruitment was done by social workers and psychologists working at the shelter. Those health care workers were familiar with MBIs. Based on their own experience with mindfulness and the mental health condition of the refugee, they advised 34 refugees to attend an information session. Inclusion criteria were (1) age between 13 and 18 years; (2) no evidence of a current severe mental illness (i.e. a clinically confirmed diagnosis of depression with suicide ideation, schizophrenia, psychosis, and personality disorders, which are not indicated with mindfulness interventions in general or with a short-term group intervention); and (3) basic knowledge of Dutch. After attending the information session 13 individuals ( 5 girls) were willing to participate, the others were not interested or attending the sessions was not compatible with their other after school activities like playing 
football, going to the fitness, or attending language classes. Mean age was $15(S D=1.15)$. Of the 13 individuals who participated, 4 arrived from Afghanistan and 2 from Albania, other countries of origin were Syria, Russia, Bosnia, Angola, Pakistan, Rwanda and Somalia.

\section{Procedure}

An exploratory pre-, post-intervention within subjects design was conducted. Participants completed 2 sets of self-administered questionnaires: at baseline (1 week before the start of the MBI) and post-intervention (1 week after MBI). Participants received 20 euro as incentive. The study was approved by the ethical committee of KU Leuven, and written informed assent and consent was obtained from all participants.

The MBI program adhered to a standardized protocol developed from the MBSR (Kabat-Zinn, 2013) and MBCT manuals (Bartley, 2012; Segal et al., 2012). The program was adjusted for young refugees and content was delivered in a very concrete and explicit manner. The program consists of eight 90-min sessions which were held once a week for 8 consecutive weeks. Each session consists of short (maximum 15 minutes) guided experiential mindfulness exercises (such as body scan, breathing space, mindful yoga, walk meditation), informal exercises (such as mindful eating and mindful listening to music), sharing of experiences of these exercises, and psycho-education on the experience of stress and self-care. The program is aimed at: (1) increasing present moment awareness and recognizing that thoughts are not real and pass away; (2) teach acceptance and mindfulness as an alternative strategy for dealing with problematic thoughts and feelings, and how these may be used to facilitate values-based actions. A brief overview of the session content is given in Table 1. Meditations were recorded and a copy was given to each participant for home use. Daily home practice was strongly encouraged.

During the study period, 3 groups of 2 to 5 participants were run. The sessions took place in the shelters of Minor Ndako, situated in the cities of Aalst and Brussels. The program was provided by two certified and experienced mindfulness trainers, a man and a women. The woman is a 45-year-old clinical psychologist who received extensive training in MBCT. She had 9 years of experience as a MBI trainer at the start of the study period and provided on average five complete 8 -week programs per year for adults as well as adolescents. The man is a 64-year-old educationist and psycho-therapist. He had ten years of experience as a MBI trainer at the start of the study period and eight years of experience in delivering psychotherapy in a welfare center working with minority groups.

\section{Measures}


The International Positive and Negative Affect Schedule Short Form (I-PANAS-SF) was used to measure positive and negative affect (Thompson, 2007). The I-PANAS-SF is composed of two 5-item mood scales: one to measure positive affect (alert, inspired, determined, attentive, active) and the other to measure negativity affect (upset, hostile, ashamed, nervous, afraid). Items are scored on a 5-point Likert Scale with higher scores reflecting higher levels of positive and negative affect. The scale showed good internal consistency for NA and adequate internal consistency for PA in our study sample (NA: $\alpha=.81$; PA: $\alpha=.74)$.

Symptoms of depression were measured using the 7-item depression subscale of the Depression Anxiety Stress Scales (DASS-21-D) (Lovibond \& Lovibond, 1995). Items are scored on a 4-point scale, where higher scores indicate higher levels of symptoms of depression. The scale showed adequate internal consistency in our study sample $(\alpha=.70)$.

The Children's Impact of Events Scale (CRIES) was used to measure the response to a traumatic event (Perrin, Meiser-Stedman, \& Smith, 2005). The scale consists of 8 items, 4 items measuring intrusion (intrusive thoughts, feelings) and 4 items measuring avoidance (avoidance of situations, feelings). Items are scored on a 4point scale, where higher scores indicate higher levels of intrusion or avoidance. The scale showed good internal consistency in our study sample $(\alpha=.88)$.

To explore how the participants experienced the MBI program semi-structured individual interviews were performed. Four participants who participated in the study were interviewed one week before and one to two weeks after the intervention. The interviews lasted 30-90 minutes and took place at Minor-Ndako. All interviews were led by a master student, who did not attend any session of the MBI program.

For both the pre- and post-intervention interview an interview guideline was used. The following questions were asked: pre-intervention: "Do you experience concerns or anxieties in Belgium? What do you expect as a result after participating in the mindfulness training?"; post-intervention: "How did you experience participating in the mindfulness training? In which way did you use the exercises from the mindfulness training in your daily life? Do you have the feeling that your concerns have decreased? Do you have the feeling that your expectations about the mindfulness training have been met?”. All questions were asked in an open manner, allowing participants to speak freely about their experiences.

The participants were free to choose the language of the interviews. One participant conducted both interviews in English and the other three participants performed the interviews in Dutch. As Dutch wasn't the mother language of none of the participants, a possible language barrier was taken into account in the interview guidelines by choosing simple and singular questions. 


\section{Data Analyses}

The intervention effect was tested using paired sample t-tests. In addition we ran a 2-level multilevel model with time-points (Level-1) nested within persons (Level-2). This allowed us to take into account the missing data and individual differences. We ran direct slope models to test whether there was a significant linear change on average before (T1) and after (T2) the intervention. The model was specified as:

$$
Y_{i j}=\beta_{0 j}+\beta_{1 j} * T_{i j}+r_{i j}
$$

where $Y_{i j}$ represents the outcome of the $j$-th participant at the $i$-th assessment time. $r_{i j}$ represents the level 1 or measurement occasion residual variance. The intercept $\left(\beta_{0 \mathrm{j}}\right)$ and slope $\left(\beta_{1 \mathrm{j}}\right)$ were allowed to vary randomly across persons. The "lme4" package (Bates, Maechler, Bolker, \& Walker, 2015) in R (Team, 2007) was used for the multilevel analysis.

Within-group effect sizes were calculated using Hedges's $g_{a v}$ which incorporates the correlation between measurements and provides a more accurate estimate in small samples (Lakens, 2013).

The semi-structured individual interviews were audio-taped and transcribed verbatim, which means that a literal reproduction of what has been said was made for each interview. After transcription the interviews were coded in the software program Nvivo 11. An open coding approach was used with codes derived directly from the data (inductive coding). This involved coding transcripts for relevant information by labeling specific sentences or short passages. A separate code list for both the pre- and post-intervention interview was created. These code lists were then used in a thematic analysis. Codes with significant overlap were merged and interview fragments moved making mutual relations between the codes visible. Emerging themes were based on both the pre- and post-intervention interviews.

From the identified codes and themes, a within-case analysis and cross-case analysis were drafted. The within-case analysis consisted of a thick description of the individual experience of each participant. Each within-case analysis was built on the same themes which were selected based on the previous encoding process. The subsequent cross-case analysis focused on general patterns across the cases, through which both similarities and differences between the cases have been identified. In the cross-case analysis themes were composed across the participants concerning the role and the experience of the MBI program.

\section{Results}




\section{Preliminary analyses}

Of the 13 individuals who participated in the MBI, 4 dropped out and did not complete the training program. The four participants who prematurely ended the training were boys, aged between 14 and 18; two were from Afghanistan, one from Albania, and one from Somalia. They did not significantly differ in any of the baseline characteristics from the other participants. Correlations among variables at baseline are given in Table 2. Gender was correlated with symptoms of depression and impact of events, indicating higher symptoms for girls compared to boys. Age was uncorrelated with outcomes. Symptoms of depression were positively associated with negative affect and impact of events. Negative affect was also positively associated with impact of events.

\section{Quantitative results}

Table 3 presents the detailed descriptive statistics of the variables of interest at baseline and postintervention. There was a significant decrease in negative affect $(t=2.49 ; p=0.04)$ with a medium effect size (.79). There was also a decrease in symptoms of depression with a large effect size (.81), however, these changes did not reach significance $(t=2.07 ; p=0.07)$. In addition there was an increase in positive affect with a medium effect size (.71), again these differences in pre- and post-scores didn’t reach significance $(t=-2.12 ; p=0.07)$. There was no difference in the pre- and post-scores for the impact of events.

In addition to the paired sample t-tests we also ran a multilevel model to test the intervention effect. This allowed us to take into account the missing data and individual differences. Table 4 presents the results from the multilevel analysis. The fixed effects of the models indicated that there was a significant decrease in symptoms of depression $(B=-3.83, p=0.046)$ and negative affect $(B=-4.16, p=0.026)$. Positive affect was significantly improved after the intervention $(B=3.09, \mathrm{p}=0.037)$.

\section{Qualitative results}

From the four participants who were interviewed only two have completed the MBI program. One participant dropped out after one session and another participant participated in only two sessions. Nevertheless, all participants were interviewed before and after the MBI program and all interviews were analysed to be able to get a complete picture of all experiences.

The within-case analysis and the subsequent cross-case analysis resulted in three main themes: Expectations regarding the MBI program, experiences of helping and non-helping factors during the MBI program and the integration of mindfulness in the coping strategies of the participants. These themes will be discussed more in-depth in what follows. 
Expectations: Reducing stress and trauma-related symptoms and social integration. Expectations related to the orientation on trauma recovery. Three participants indicated how they expected a reduction in different stress- \& trauma-related symptoms by participating. They expressed that participating to the MBI program will help them to reduce symptoms as a tense functioning, sleep disorders, arousal or aggressive behavior. Other participants also described how they expect to learn to regain control about their emotions and thoughts.

I would like to sleep better. Not dreaming with bad things anymore. I hope to improve a lot. Maybe I will be able to use the exercises.

I expect that if I'm angry I know how to control myself. I would like to have more control. Sometimes it is ok, but sometimes I have more control than before, but I want even more control over myself when I'm angry.

Expectations equally related to the hope of social integration. Two participants mentioned how their participation was related to their hope to engage in more social contacts. They expected that the contact with other participants would be helpful in learning to deal with their concerns. One participant also described the expectation that the MBI program will offer more knowledge about the host country which will make it able to integrate more easily. It's good to be with others. Then you really want to participate. With friends is more fun. Imagine I was alone, that would not be fun. Then I can't talk to anyone.

\section{Experiences: Concerns about the format, duration and language of the MBI program. Helping} and non-helping factors related to engaging in social relationships during the MBI program. Due to the fact that two of the four participants did not complete the training the MBI group consisted of only two participants. One participant described how this absence of interaction with other participants was a non-helping factor. The participant felt a lack of social support during the MBI program.

We were only two persons. Sometimes I was alone. Normally I like to be in a group. When I was alone, I was just, I was not able to think anymore.

Helping and non-helping factors also related to specific intervention characteristics. Different participants mentioned how the language of the MBI program has hampered an adequate participation. One of these participants experienced problems in performing correctly the exercises. However, both participants mention how this language barrier seemed to be slightly reduced by the possibility to interact with the trainer. Furthermore, this interaction made it possible for the participants to obtain additional information about the concepts and attitudes of the practice of mindfulness, which they experienced as helpful. 
In the training she [trainer] told us what mindfulness was about. Living in the present is everything and the training really learned me to focus on what I have and where I am right now.

Other factors related to the specific intervention characteristics were the duration of the sessions, the perception of the mindfulness techniques and the use of the techniques in their daily life. Different participants indicated that the duration of the sessions was too long which made it hard to concentrate. This was also one of the reasons why one of the two participants stopped after following two sessions.

It takes long time, one hour or more. It is boring. If something is not interesting you don't want to stay, you want to go. For me it was difficult to concentrate and nothing changed between the first and second session.

The other participant who dropped expressed a lack of alignment between personal functioning and the intervention.

I went to one session, I see the exercise and it was kind of interesting. We had to think and this was good. It was not difficult but I don't need it. This is just not for me.

Of the two participants who completed the training one participant practiced during the MBI, the other participant did not practice because of a lack of time or because she forgot it.

To practice the exercises was quiet difficult to do because I barely did it on my own so. It was just something that didn't cross my mind. I didn't think about practicing.

Both participants mentioned the use of the exercises in their daily life after the intervention. One participant indicated that he preferred to use the short exercises ( 5 minutes) in his daily life instead of the longer exercises (10-15 minutes).

When I can't concentrate at school or when I go to sleep at night, I do an exercise. It helps.

\section{Integration: Mindfulness exercises used as a new coping strategy in combination with familiar}

coping strategies. The two participants who completed the training mentioned that after the MBI program they made use of familiar or cultural coping strategies, such as religious affiliation and practices, sport, or active silencing (Kevers, Rober, Derluyn, \& De Haene, 2016). Here, participants used mindfulness techniques as novel coping strategies, implying their process of combining cultural strategies and new coping strategies.

I do mindfulness exercises and I also do other things, such as praying and sport. The mindfulness exercises are helpful when I can't concentrate with school or when I have a lot of stress. I do some exercises. At night I prefer to pray. 
Running head: MINDFULNESS FOR UNACCOMPANIED REFUGEE MINORS

Interestingly, one participant indicated how using mindfulness techniques may closely align with cultural strategies of active silencing, through enabling her focus on the present.

I analyze less. I started to analyze less during the training. I became nicer. I realized during the training that I have to live now. So I stopped with analyzing everything and I just live and I enjoy life.

\section{Discussion}

The purpose of this pilot study was to explore the feasibility of MBI for unaccompanied foreign minors residing at the shelters of Minor Ndako. Our preliminary findings suggest that MBI may be effective in reducing symptoms of negative effect and improving positive affect, both with a medium effect size, and in reducing symptoms of depression with a large effect size. However, the changes for symptoms of depression and positive affect cannot claim statistical significance due to the small sample size.

Although this study was undertaken in a new context and different population, these preliminary findings are consistent with previous MBI intervention research (Gotink et al., 2015; Khoury et al., 2014). The initial results are also in line with MBI theory research suggesting that mindfulness skills increase the ability of the individual to handle the thoughts and emotions that they experience via an increased awareness and acceptance, as opposed to a trying to escape or avoid them (Briere, 2012; Kuyken et al., 2010). However, we have to interpret these preliminary quantitative results with great caution due to the small self-selected sample and the lack of a control-group. We cannot rule out that unspecific effects such as social support and the weekly attention of two trainers may explain a significant proportion of our results, nor can we rule out that the observed effects are a simple effect of the mere passage of time. These initial quantitative findings therefore only indicate that MBIs may be useful in this unique context and population. Further research is needed to replicate and further extend these preliminary results.

Our results are also in line with previous studies indicating that interventions, offered in group format are helpful in alleviating mental health problems among refugees (Behnia, 2003; Kalmanowitz, 2016; Mitschke, Praetorius, Kelly, Small, \& Kim, 2017; Quinlan, Schweitzer, Khawaja, \& Griffin, 2016). Mitschke et al. (2017) interviewed 30 refugees who had received different kinds (individual and group structure) of treatment for depression, PTSD, or anxiety. The overall consensus of participants who reflected on the program's efficacy appreciated the group structure over the individual counseling model. The favor for the group model had to do with opportunities for group support (interconnectedness) and psycho-education and with perceptions of distrust, 
stigma and uncertainty about the formal, individualized counseling services. Refugees sometimes perceive talking therapy as distressing and intrusive.

Our qualitative findings show that the expectations of the participants prior to the MBI program together with their will and hope for a better future, the guidance to the MBI program and the advice from the care workers in the shelter, seem to be important elements in their decision to participate to the MBI program. Although four participants had the intention to participate only two of them completed the training. This shows how unique and different the experiences of the participants are. The main reasons for not attending the whole training were lack of interest and experiencing the first sessions as boring. The two participants who completed the training experienced MBI as helpful and make use of the mindfulness exercises as a new coping strategy in combination with sport and other familiar coping strategies. Familiar coping strategies that were mentioned frequently among the participants were the use of religion, isolating oneself from others and deliberately silence about traumatic experiences in the past (Kevers et al., 2016). Other studies with UMs also mentioned these coping strategies as frequently used strategies in the host country (Carlson, Cacciatore, \& Klimek, 2012; Derluyn, Wille, De Smet, \& Broekaert, 2005; Hornor, 2013). Both participants expressed concerns about the format, the duration of the sessions was experienced as too long, and language of the intervention.

Reflecting on the challenges of providing MBIs to UMs, trainers indicated that practical- and languagerelated factors were the most challenging difficulties. Practical difficulties were mainly due to time-table incompatibilities with after-school activities like sport, social activities. This is also in line with our previous research involving adolescents and young adults (Van der Gucht et al., 2017b). Adolescents have a very busy life and are very active and a commitment to follow 8 weekly sessions is often the mean reason for non-participating. Therefore the arrangement of 8 weekly sessions might need to be revised for the future. We think that the logical next step would be to run a revised version of the training within a larger study.

\section{Strengths and limitations}

A strength is that quantitative findings were presented with qualitative findings to provide an overview of the effects and experiences of MBI participation. A limitation is that the quantitative study was completed with final data on only 13 participants and the qualitative results were based on only 4 participants. There are several reasons for that. First, the recruitment number ( $38 \%$ of the individuals who attended the information session) was low and attrition rate was high (30\% at post-intervention). A recruitment number of $38 \%$ is not unusual in trials with adolescents and young adults (Burke, Albritton, \& Marina, 2007). Difficulties with 
recruitment in this kind of trials is a common problem and, it is not unique to this specific context. A drop-out rate of 30\% at post-intervention is partly due to practical and organizational problems, weekly sessions, duration of the sessions, and language problems. These issues need to be addressed in future studies to enlarge treatment acceptability. Second, a lack of financial support meaning that we could only take a limited number of qualitative interviews in the framework of a master thesis. No specific recruitment strategy was used for qualitative study participants. Because of these limitations saturation was not part of the qualitative methodology, and inter-coder reliability was not available. We also cannot ensure that prior concerns before moving to Belgium are motivating the presentation of concerns now. Third, organizational issues that are inevitable with this kind of trial had to be taken care of by the people of vzw Minor Ndako on top of their regular daily work. Fourth, the timing of the study: During the study period the shelter was overwhelmed by new, mostly very young, refugees due to the refugee crisis (March 2015-March 2016) and there was a change in the chair of vzw MinorNdako.

More robust high quality trials are needed to see whether the results can be replicated. Outcomes could have been achieved through trainer commitment or group effect. Future research should disentangle specific and non-specific factors to clarify effectiveness utilizing active control conditions. Long term follow-up data were missing due to the limited stay in Minor-Ndako. We further have to take into account a potential risk of bias due to the real-time non-standardized translation of assessment measures.

Funding

This study was funded by a grant from the foundation "Stichting Koningin Paola". The writing of this article has been facilitated by KU Leuven Center for Excellence on Generalization Research (GRIP*TT; PF/10/005).

\section{Conflict of Interest}

The authors declare no conflict of interest.

\section{Ethical approval}

All procedures performed in studies involving human participants were in accordance with the ethical standards of the institutional and/or national research committee and with the 1964 Helsinki declaration and its later 
Running head: MINDFULNESS FOR UNACCOMPANIED REFUGEE MINORS

amendments or comparable ethical standards. The study was approved by the ethics committee of KU Leuven on $7^{\text {th }}$ November 2014 (SMEC G-2014 10 91).

Informed Consent

Informed assent and consent was obtained from all individual participants included in the study.

\section{Author Contributions}

KVDG: designed and executed the study, analyzed the quantitative data, and wrote the paper. JG: collected and analyzed the qualitative data and collaborated with the writing of the study. LDH: collaborated with the qualitative analyses and writing. PK and FR: collaborated with the design, writing and editing of the final manuscript. 


\section{References}

Acarturk, C., Konuk, E., Cetinkaya, M., Senay, I., Sijbrandij, M., Gulen, B., \& Cuijpers, P. (2016). The efficacy of eye movement desensitization and reprocessing for post-traumatic stress disorder and depression among Syrian refugees: results of a randomized controlled trial. Psychological Medicine, 46(12), 25832593.

Bartley, T. (2012). Mindfulness-based cognitive therapy for cancer. West-Sussex: John Wiley \& Sons Ltd.

Bates, D., Maechler, M., Bolker, B., \& Walker, S. (2015). Fitting Linear Mixed-Effects Models Using lme4. Journal of Statistical Software, 67(1), 1-48. doi:10.18637/jss.v067.i01

Bean, T. M., Eurelings-Bontekoe, E., \& Spinhoven, P. (2007). Course and predictors of mental health of unaccompanied refugee minors in the Netherlands: One year follow-up. Social Science \& Medicine, 64(6), 1204-1215.

Behnia, B. (2003). Refugees' convoy of social support: Community peer groups and mental health services. International Journal of Mental Health, 32(4), 6-19.

Briere, J. (2012). Working with trauma: Mindfulness and compassion. In C.K. Germer \& R.D. Siegel (Eds), Wisdom and Compassion in psychotherapy. New York, The Guilford Press.

Burke, M. E., Albritton, K., \& Marina, N. (2007). Challenges in the recruitment of adolescents and young adults to cancer clinical trials. Cancer, $110(11), 2385-2393$.

Carlson, B. E., Cacciatore, J., \& Klimek, B. (2012). A risk and resilience perspective on unaccompanied refugee minors. Social work, 57(3), 259-269.

Catani, C., Kohiladevy, M., Ruf, M., Schauer, E., Elbert, T., \& Neuner, F. (2009). Treating children traumatized by war and Tsunami: a comparison between exposure therapy and meditation-relaxation in North-East Sri Lanka. BMC Psychiatry, 9(1), 22.

Crane, R., Brewer, J., Feldman, C., Kabat-Zinn, J., Santorelli, S., Williams, J., \& Kuyken, W. (2017). What defines mindfulness-based programs? The warp and the weft. Psychological medicine, 47(6), 990-999.

Derluyn, I., \& Broekaert, E. (2008). Unaccompanied refugee children and adolescents: The glaring contrast between a legal and a psychological perspective. International journal of law and psychiatry, 31(4), 319-330.

Derluyn, I., Mels, C., \& Broekaert, E. (2009). Mental health problems in separated refugee adolescents. Journal of Adolescent Health, 44(3), 291-297. 
Derluyn, I., Wille, B., De Smet, T., \& Broekaert, E. (2005). Op weg. Psychosociale en therapeutische begeleiding van niet-begeleide buitenlandse minderjarigen. Antwerpen: Garant.

Fazel, M., Reed, R. V., Panter-Brick, C., \& Stein, A. (2012). Mental health of displaced and refugee children resettled in high-income countries: risk and protective factors. The Lancet, 379(9812), 266-282.

Fazel, M., Wheeler, J., \& Danesh, J. (2005). Prevalence of serious mental disorder in 7000 refugees resettled in western countries: a systematic review. The Lancet, 365(9467), 1309-1314.

Gotink, R. A., Chu, P., Busschbach, J. J., Benson, H., Fricchione, G. L., \& Hunink, M. M. (2015). Standardised mindfulness-based interventions in healthcare: an overview of systematic reviews and meta-analyses of RCTs. Plos One, 10(4), e0124344.

Gutner, C. A., Galovski, T., Bovin, M. J., \& Schnurr, P. P. (2016). Emergence of transdiagnostic treatments for PTSD and posttraumatic distress. Current Psychiatry Reports, 18(10), 95.

Hinton, D. E., Ojserkis, R. A., Jalal, B., Peou, S., \& Hofmann, S. G. (2013). Loving-Kindness in the Treatment of Traumatized Refugees and Minority Groups: A Typology of Mindfulness and the Nodal Network Model of Affect and Affect Regulation. Journal of clinical psychology, 69(8), 817-828. doi:10.1002/jclp.22017

Hinton, D. E., Pich, V., Hofmann, S. G., \& Otto, M. W. (2013). Acceptance and Mindfulness Techniques as Applied to Refugee and Ethnic Minority Populations With PTSD: Examples From "Culturally Adapted CBT". Cognitive and Behavioral Practice, 20(1), 33-46.

Hornor, G. (2013). Posttraumatic stress disorder. Journal of Pediatric Health Care, 27(3), e29-e38.

Huemer, J., Karnik, N. S., Voelkl-Kernstock, S., Granditsch, E., Dervic, K., Friedrich, M. H., \& Steiner, H. (2009). Mental health issues in unaccompanied refugee minors. Child and adolescent psychiatry and mental health, 3(1), 13.

Jalal, B., Samir, S. W., \& Hinton, D. E. (2017). Adaptation of CBT for Traumatized Egyptians: Examples from Culturally Adapted CBT (CA-CBT). Cognitive and Behavioral Practice, 24(1), 58-71.

Kabat-Zinn, J. (1994). Wherever you go there you are: mindfulness meditation in everyday life. New York: Hyperion.

Kabat-Zinn, J. (2013). Full catastrophe living, revised edition: how to cope with stress, pain and illness using mindfulness meditation. London: Piatcus.

Kalmanowitz, D. (2016). Inhabited studio: Art therapy and mindfulness, resilience, adversity and refugees. International Journal of Art Therapy, 21(2), 75-84. 
Kalmanowitz, D., \& Ho, R. T. (2016). Out of our mind. Art therapy and mindfulness with refugees, political violence and trauma. The Arts in Psychotherapy, 49, 57-65.

Kevers, R., Rober, P., Derluyn, I., \& De Haene, L. (2016). Remembering collective violence: Broadening the notion of traumatic memory in post-conflict rehabilitation. Culture, medicine, and psychiatry, 40(4), 620-640.

Khoury, B., Lecomte, T., Fortin, G., Masse, M., Therien, P., Bouchard, V., . . Hofmann, S. G. (2014). Mindfulness-based therapy: A comprehensive meta-analysis. Clinical Psychology Review, 33(6), 763771. doi:10.1016/j.cpr.2013.05.005

Khoury, B., Sharma, M., Rush, S. E., \& Fournier, C. (2015). Mindfulness-based stress reduction for healthy individuals: A meta-analysis. Journal of Psychosomatic Research, 78(6), 519-528. doi:10.1016/j.jpsychores.2015.03.009

Kuyken, W., Warren, F. C., Taylor, R. S., Whalley, B., Crane, C., Bondolfi, G., . . Dalgleish, T. (2016). Efficacy of Mindfulness-Based Cognitive Therapy in Prevention of Depressive Relapse An Individual Patient Data Meta-analysis From Randomized Trials. JAMA psychiatry, 73(6), 565-574. doi:10.1001/jamapsychiatry.2016.0076

Kuyken, W., Watkins, E., Holden, E., White, K., Taylor, R. S., Byford, S., . . Dalgleish, T. (2010). How does mindfulness-based cognitive therapy work? Behaviour Research and Therapy, 48(11), 1105-1112. doi:10.1016/j.brat.2010.08.003

Lakens, D. (2013). Calculating and reporting effect sizes to facilitate cumulative science: a practical primer for ttests and ANOVAs. Frontiers in Psychology, 4, 863.

Lang, A. J. (2017). Mindfulness in PTSD treatment. Current Opinion in Psychology, 14, 40-43. doi:10.1016/j.copsyc.2016.10.005

Lovibond, S. H., \& Lovibond, P. F. (1995). Manual for the depression anxiety stress scales (2nd ed.). Sidney: Psychology Foundation.

Lustig, S. L., Kia-Keating, M., Knight, W. G., Geltman, P., Ellis, H., Kinzie, J. D., . . Saxe, G. N. (2004). Review of child and adolescent refugee mental health. Journal of the American Academy of Child \& Adolescent Psychiatry, 43(1), 24-36.

Miller, K. E., \& Rasmussen, A. (2010). War exposure, daily stressors, and mental health in conflict and postconflict settings: bridging the divide between trauma-focused and psychosocial frameworks. Social Science \& Medicine, 70(1), 7-16. 
Mitschke, D. B., Praetorius, R. T., Kelly, D. R., Small, E., \& Kim, Y. K. (2017). Listening to refugees: How traditional mental health interventions may miss the mark. International social work, 60(3), 588-600.

Neuner, F., Onyut, P. L., Ertl, V., Odenwald, M., Schauer, E., \& Elbert, T. (2008). Treatment of posttraumatic stress disorder by trained lay counselors in an African refugee settlement: a randomized controlled trial. Journal of Consulting and Clinical Psychology, 76(4), 686-694.

Neuner, F., Schauer, M., Klaschik, C., Karunakara, U., \& Elbert, T. (2004). A comparison of narrative exposure therapy, supportive counseling, and psychoeducation for treating posttraumatic stress disorder in an african refugee settlement. Journal of Consulting and Clinical Psychology, 72(4), 579.

Nosè, M., Ballette, F., Bighelli, I., Turrini, G., Purgato, M., Tol, W., . . Barbui, C. (2017). Psychosocial interventions for post-traumatic stress disorder in refugees and asylum seekers resettled in high-income countries: Systematic review and meta-analysis. Plos One, 12(2), e0171030.

Perrin, S., Meiser-Stedman, R., \& Smith, P. (2005). The Children's Revised Impact of Event Scale (CRIES): Validity as a screening instrument for PTSD. Behavioural and Cognitive Psychotherapy, 33(4), 487.

Porter, M., \& Haslam, N. (2005). Predisplacement and postdisplacement factors associated with mental health of refugees and internally displaced persons: a meta-analysis. Jama, 294(5), 602-612.

Quinlan, R., Schweitzer, R. D., Khawaja, N., \& Griffin, J. (2016). Evaluation of a school-based creative arts therapy program for adolescents from refugee backgrounds. The Arts in Psychotherapy, 47, 72-78.

Segal, Z. V., Williams, J. M. G., \& Teasdale, J. D. (2012). Mindfulness-based cognitive therapy for depression. New York: Guilford Press.

Spijkerman, M., Pots, W., \& Bohlmeijer, E. (2016). Effectiveness of online mindfulness-based interventions in improving mental health: A review and meta-analysis of randomised controlled trials. Clinical Psychology Review, 45, 102-114.

Team, R. D. C. (2007). R: A Language and Environment for Statistical Computing Vienna: R Foundation for Statistical Computing.

Thompson, E. R. (2007). Development and validation of an internationally reliable short-form of the positive and negative affect schedule (PANAS). Journal of cross-cultural psychology, 38(2), 227-242.

Tol, W. A., Barbui, C., Galappatti, A., Silove, D., Betancourt, T. S., Souza, R., . . Van Ommeren, M. (2011). Mental health and psychosocial support in humanitarian settings: linking practice and research. The Lancet, 378(9802), 1581-1591. 
Van der Gucht, K., Takano, K., Kuppens, P., \& Raes, F. (2017). Potential Moderators of the Effects of a SchoolBased Mindfulness Program on Symptoms of Depression in Adolescents. Mindfulness, 8(3), 797-806. doi:10.1007/s12671-016-0658-X

Van der Gucht, K., Takano, K., Labarque, V., Vandenabeele, K., Nolf, N., Kuylen, S., . . Raes, F. (2017). A Mindfulness-Based Intervention for Adolescents and Young Adults After Cancer Treatment: Effects on Quality of Life, Emotional Distress, and Cognitive Vulnerability. Journal of Adolescent and Young Adult Oncology, 6(2), 307-317.

Van der Gucht, K., Takano, K., Raes, F., \& Kuppens, P. (2018). Processes of change in a school-based mindfulness programme: cognitive reactivity and self-coldness as mediators. Cognition and Emotion, 32(3), 658-665.

Vervliet, M., Lammertyn, J., Broekaert, E., \& Derluyn, I. (2014). Longitudinal follow-up of the mental health of unaccompanied refugee minors. European Child \& Adolescent Psychiatry, 23(5), 337-346.

Vuković, I. S., Jovanović, N., Kolarić, B., Vidović, V., \& Mollica, R. F. (2014). Psychological and somatic health problems in Bosnian refugees: A three year follow-up. Psychiatria Danubina, 26(3), 306-444. 
Table 1. Core elements of the 8-week mindfulness-based intervention

\begin{tabular}{|l|l|}
\hline Session & Content \\
\hline 1 & $\begin{array}{l}\text { Observing the automatic pilot and kind attention to the body } \\
\text { Brief grounding exercise, Spotlight metaphor of attention, Ground rules, Intentions, Pause } \\
\text { exercise, Eating exercise, Body Scan }\end{array}$ \\
\hline 2 & $\begin{array}{l}\text { Gently dealing with barriers while practicing and befriending the breath } \\
\text { Body Scan, Monkey-mind metaphor of thoughts, Pause exercise, Breath as the anchor exercise, } \\
\text { Walking meditation }\end{array}$ \\
\hline 3 & $\begin{array}{l}\text { Gently learning to work with personal limits and befriending the breath } \\
\text { Mindful movement, Mindful walking, exercise on entanglement of thoughts-feelings-sensations, } \\
\text { 3-minute breathing space }\end{array}$ \\
\hline 5 & $\begin{array}{l}\text { Discovering that we can choose how to respond by opening gently to experience } \\
\text { Sitting meditation (body, breath, sound), Inhibition exercise, Pause and 3-minute breathing space } \\
\text { as responding tool }\end{array}$ \\
\hline 6 & $\begin{array}{l}\text { Gently being with what is difficult } \\
\text { Sitting meditation (body, breath, difficult feelings), Body Scan, Pause and Barometer exercise, }\end{array}$ \\
\hline 7 & $\begin{array}{l}\text { Learning to accept that thoughts are not facts } \\
\text { Sitting meditation (body, breath, sounds, thoughts), Gently experiencing the power of thoughts, } \\
\text { Mountain meditation, Barometer exercise }\end{array}$ \\
\hline 8 & $\begin{array}{l}\text { Taking care of ourselves } \\
\text { Body scan (standing), Spiral signature and generating a personal list of practices, Mindful } \\
\text { movement, Barometer exercise }\end{array}$ \\
\hline $\begin{array}{l}\text { Going further, beyond fear } \\
\text { Pause and Barometer, Body scan, Reviewing personal action plans, Walking meditation, Review } \\
\text { course, Loving kindness meditation }\end{array}$ \\
\hline
\end{tabular}


Running head: MINDFULNESS FOR UNACCOMPANIED REFUGEE MINORS

Table 2. Correlations among variables at baseline (T1)

\begin{tabular}{l|lrrrrr} 
& \multicolumn{1}{|c}{1} & \multicolumn{1}{c}{2} & \multicolumn{1}{c}{3} & 4 & 5 & 6 \\
\hline 1. Gender & 1.000 & & & & & \\
2. Age & -0.291 & 1.000 & & & & \\
3. DASS-D & $0.715^{* *}$ & 0.085 & 1.000 & & & \\
4. PA & 0.219 & -0.103 & 0.029 & 1.000 & & \\
5. NA & 0.516 & 0.293 & $0.648^{*}$ & -0.405 & 1.000 & \\
6. IES & $0.576^{*}$ & 0.098 & $0.808^{* *}$ & 0.056 & $0.704 * *$ & 1.000 \\
\hline
\end{tabular}

*p<.05, **p<.01; DASS-D, symptoms of depression; PA, Positive Affect; NA, Negative Affect; IES, Impact of Events 
Running head: MINDFULNESS FOR UNACCOMPANIED REFUGEE MINORS

Table 3. Outcome scores at each assessment point and effect sizes

\begin{tabular}{l|ccc}
\hline & T1 & T2 & Hedges's $g_{a v}$ \\
& M (SD) & M (SD) & \\
\hline Symptoms of depression & 10.89 & 6.89 & 0.81 \\
Negative Affect & $(4.78)$ & $(4.17)$ & \\
Positive Affect & 16.00 & 11.56 & 0.79 \\
& $(6.50)$ & $(3.00)$ & \\
Impact of Events & 15.00 & 18.00 & 0.71 \\
& $(4.24)$ & $(3.84)$ & \\
& 22.38 & 21.88 & 0.05 \\
\hline
\end{tabular}


Running head: MINDFULNESS FOR UNACCOMPANIED REFUGEE MINORS

Table 4. Estimates from the multilevel models

\begin{tabular}{l|ll} 
& Intercept & $\boldsymbol{B}$ (SE) \\
\hline DASS-D & $10.69(1.17)$ & $-3.83(1.71)^{*}$ \\
& & $\mathrm{P}=.046$ \\
PA & $14.85(0.99)$ & $3.09(1.28)^{*}$ \\
& & $\mathrm{P}=.037$ \\
NA & $15.54(1.28)$ & $-4.16(1.62)^{*}$ \\
& & $\mathrm{P}=.026$ \\
IES & $23.46(2.53)$ & $-1.08(3.16)$ \\
& & $\mathrm{P}=.74$ \\
\hline
\end{tabular}

$* \mathrm{p}<.05$ 